\title{
Application of Multivariable Feedback Methods to Intravenous Anesthetic Pharmacodynamics
}

\author{
Geir Dullerud \\ Department of Electrical Engineering \\ California Institute of Technology \\ Pasadena, CA91125
}

\author{
Marie Csete \\ Department of Anesthesiology \\ University of California \\ Los Angeles, CA90095-1778
}

\author{
John Doyle \\ Department of Electrical Engineering \\ California Institute of Technology \\ Pasadena, CA91125
}

\begin{abstract}
Continuous infusions of intravenous anesthetics are becoming increasingly popular during surgical procedures, largely because relatively precise, consistent control of anesthetic depth is possible over intravenous injection techniques. In this paper we investigate the main issues involved with the development of automatic intravenous anesthesia delivery systems in the context of robust multivariable control. We present a pharmacodynamic model that may be suitable for closed-loop control, and discuss clinical data collected from human subjects during actual surgical conditions with the anesthetic propofol.
\end{abstract}

\section{Introduction}

This paper considers automatic control of anesthetic depth during surgery with a view to applying multivariable control methods. A preliminary investigation is conducted into modeling and control for automated anesthesia delivery, which includes analysis of clinical data collected during surgery.

Continuous infusions, delivered by digitally programmed pumps, are a commonly used approach for delivering intravenous anesthetic during surgical procedures. They allow the physician to precisely set the flow rate of anesthetic into the patient in an attempt to maintain appropriate levels of anesthetic depth. This method of administering drug has largely replaced the previous approach in which, instead, a series of periodic injections are given. The advantage of the infusion method is that the drug level in the body can be kept more constant, in contrast to the sequence of peaks and valleys that result from periodic injections - thus less drug is needed, increasing patient safety.

Although such infusion systems enable physicians to continuously adjust the rate of anesthetic up-

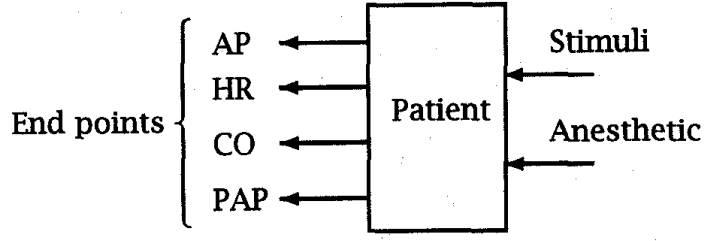

Figure 1: Anesthetic Model

take, it has been suggested in the medical literature [4][7][9] that this task could be more accurately performed by an automatic feedback control device. Firstly, because during routine surgical situations automatic control of the anesthetic delivery would free the clinician to focus on more important issues. Secondly, because a control system can continuously monitor and make fine adjustments, so that the minimum amount of drug required to achieve a desired effect is used.

We hope that the issues raised in this paper will provide the basis for a program of successful development of automated intravenous anesthesia delivery.

\section{Anesthesia Background}

The main objective of anesthesia during surgery is to ensure that the patient is sufficiently insensitive to painful stimuli. At the same time, it is important for safety reasons, that the patient not be overly anesthetized. Unfortunately, so far, physicians do not have a direct and reliable method to measure the degree of patient mental awareness or anesthetic depth. In particular, and contrary to popular intuition, EEG signals alone do not provide an accurate indication of anesthetic depth.

The approach that is adopted for estimating the ill-defined quantity anesthetic depth is to focus on physical processes that are directly regulated by 


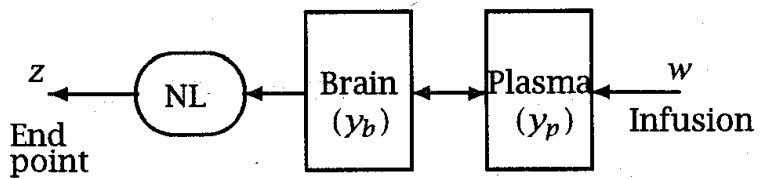

Figure 2: Pharmacodynamic Model

the brain. Physicians controlling anesthetic depth take an input-output view of the patient as shown in Figure 1. The inputs to the patient are the physical stimuli and the dose of anesthetic. The outputs, or end points, are measurements such as arterial blood pressure (AP), heart rate (HR), cardiac output $(\mathrm{CO})$ and pulmonary arterial pressure (PAP). The key property of each of these end points is that they are controlled by the brain. Thus their levels, to some degree, reflect the perception of painful stimuli. During surgery, if these endpoints react significantly to the stimuli that are applied to the patient, such as an incision to the skin, it is concluded that the anesthetic depth is insufficient and more anesthetic is required.

Maintaining a particular anesthetic depth, in the absence of stimuli, therefore translates into keeping these endpoints at a constant level, which is the goal of an automatic control system for control of intravenous anesthesia. Thus, a model is required that relates the endpoints to the timehistory of the anesthetic input - namely a pharmacodynamic model.

\section{Pharmacodynamic Modeling}

Pharmacodynamic models have long been of interest in medicine and pharmacology. Despite this history, there are few models that enjoy substantial predictive success [3]. This is due in part to the range of variation between human subjects, but is largely because the precise mechanisms by which most anesthetic drugs operate are not well understood.

Fortunately, we seek a model for the purposes of closed-loop, or feedback, control. Thus a simple model that captures the salient dynamical features of the pharmacodynamic process is both sufficient and preferred. The structure of the model we propose is shown in Figure 2. Models of a similar form appear in various versions in the literature (see bibliographic references), and we believe the one presented here may be particularly suitable for control.
The model consists of three dynamical components: the first is the plasma into which the drug infusion flows; the plasma interacts with the brain through the blood brain barrier so that part of the drug entering the plasma eventually enters the brain; the brain sets the end point, which depends on the drug concentration in the brain.

The models we choose for the plasma and brain concentrations, $y_{p}$ and $y_{b}$ respectively, ignoring for now their interaction, are linear time-invariant systems corresponding to the transfer functions

$$
\begin{aligned}
& T_{p}(s):=\frac{A}{s+a}+\frac{B}{s+b}+\frac{C}{s+c} \\
& T_{b}(s):=\frac{D}{s+d}
\end{aligned}
$$

where all the parameters are real and positive. These transfer functions can be associated with the standard three- and one- compartment models (see e.g., [3][4]).

The interaction between the brain and plasma components of the model are described by the linear gradient equation

$$
\text { flow }(t)=E\left(y_{p}(t)-y_{b}(t)\right)
$$

where $E$ is a non-zero parameter. This equation can be interpreted as flow through a membrane. Hence, the map from the infusion $w$ to $y_{b}$ can be described by a stable transfer function of the form

$$
T(s)=\frac{a_{2} s^{2}+a_{1} s+a_{0}}{s^{4}+b_{3} s^{3}+b_{2} s^{2}+b_{1} s+b_{0}} .
$$

The final component of the model is comprised of a time delay and a static nonlinearity. Given a parametrized family of functions, $\mathcal{F}$, from which the nonlinearity is chosen, the map from $y_{b}$ to the endpoint $z$ is defined by

$$
z(t)=F\left(y_{b}\left(t-t_{0}\right)\right)
$$

where $F \in \mathcal{F}$ and $t_{0}>0$. This is the least physically motivated of the components. The simplest relevant choice for the family $\mathcal{F}$ is the set of oneparameter affine functions; in [8][3] a set of sigmoidal curves is chosen. The time delay is also an empirically determined part of the model, and is clearly necessary as we see in the next section. Some have suggested that this delay is due to recirculation time ([5]) in the plasma, but this is not conclusive.

The parameters in (2) and the chosen set $\mathcal{F}$ are expected to vary significantly among individual 
subjects as is commonly observed in pharmacodynamic and pharmacokinetic models; thus the use of the above model for feedback control may require customized fitting. An important feature of (2), that does not seem to be widely appreciated, is that the transfer function has relative degree of at least two. In, e.g. [5][8], this is not the case: namely the model predicts a discontinuous jump in the end point when an injection is made; a situation that is seldom observed.

\section{Clinical Data}

This section presents data collected from three human subjects during surgery. We will not evaluate the model of the previous section here, as our data set is small and the sources of stimuli significant; a detailed evaluation will be reported elsewhere. The data is used to illustrate some of the inherent difficulties associated with control of anesthetic depth. The subjects studied were patients about to undergo liver transplant surgery at UCLA Medical Center ${ }^{1}$; the specific anesthetic used was propofol. The advantage in studying these patients is that a larger number of end points are available than in more minor surgical procedures. Of course a partial disadvantage is the (hemodynamic) responses observed may not be strongly representative of those usually observed in healthier subjects.

The endpoints we were able to measure are those listed in Figure 1. Here we do not include cardiac output since it is measured infrequently. The data was collected during the one hour period prior to the main (abdominal) incision. The plots of the (systemic) arterial pressures have two traces: systolic and diastolic pressures. When the patients were first anesthetized these pressures were measured using a noninvasive cuff around the patients arm, which produced readings at periodic intervals. Later a catheter was inserted into a radial artery which provided continuous measurement of arterial pressure. Thus the transition from sampled to continuous pressure readings in Figures 7 and 9. The data in all the plots (except the sampled cuff measurements) are low-pass filtered to smooth quantization error. The time profiles of the amounts of drug administered to the three patients are shown in Table 1; the table provides the amount of propofol given by injection (bolus), and the programmed setting changes to the continuous infusion pump.

\footnotetext{
${ }^{1}$ after approval from the UCLA Human Subjects Protection Committee
}

\begin{tabular}{|l||l|l|l|}
\hline & Pat. A & Pat. B & Pat. C \\
\hline \hline time $(\mathrm{min})$ & $0: 00$ & $1: 30$ & $4: 30$ \\
bolus $(\mathrm{mg} / \mathrm{kg})$ & 2.86 & 2 & 2.05 \\
rate $(\mu \mathrm{g} / \mathrm{kg} / \mathrm{min})$ & 100 & 100 & 100 \\
\hline time $(\mathrm{min})$ & $6: 00$ & $9: 30$ & $6: 30$ \\
rate $(\mu \mathrm{g} / \mathrm{kg} / \mathrm{min})$ & 150 & 50 & - \\
bolus $(\mathrm{mg} / \mathrm{kg})$ & - & - & 0.71 \\
\hline time $(\mathrm{min})$ & $13: 00$ & - & $10: 00$ \\
bolus $(\mathrm{mg} / \mathrm{kg})$ & 1.43 & - & 1 \\
\hline time $(\mathrm{min})$ & $25: 00$ & - & $14: 15$ \\
rate $(\mu \mathrm{g} / \mathrm{kg} / \mathrm{min})$ & 120 & - & 50 \\
\hline time $(\mathrm{min})$ & $31: 45$ & - & $25: 15$ \\
rate $(\mu \mathrm{g} / \mathrm{kg} / \mathrm{min})$ & 200 & - & 100 \\
\hline
\end{tabular}

Table 1: Propofol Time-histories

Patient A had arterial and pulmonary arterial lines in place before the start of anesthesia; these were used previously for monitoring the patient in the intensive care unit. He had a tube in his trachea and was mechanically ventilated. Figure 3 shows the heart rate of the patient from the time that anesthetic was first given. This response was atypical, since the heart rate increased (tachycardia) after propofol was administered, whereas a characteristic response is a decrease. This was interpreted as a reflex response, by the heart, due to the patient having low blood volume: propofol causes blood vessels to dilate, and therefore, if intravascular volume is low, the heart senses less blood return and responds by increasing its rate. Figure 4 plots the measured arterial pressures (systolic and diastolic) and is qualitatively typical; that is, the pressures decrease with administration of anesthetic. However, the response is unusually small.

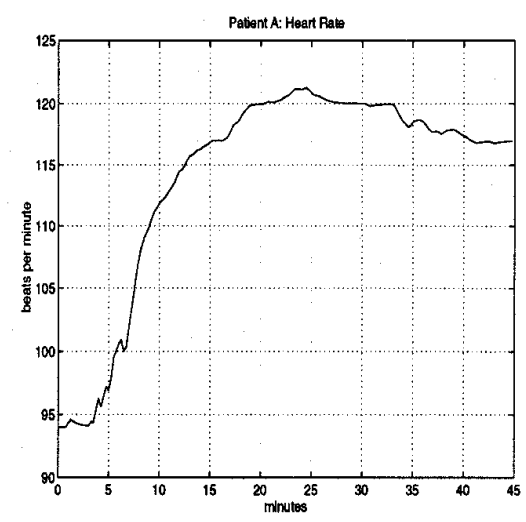

Figure 3: Patient A: Heart Rate

Pulmonary arterial pressures are shown in Figure 5: these pressures are initially higher than usual because the patient was awake and on a ventilator (which is very painful). These pressures 


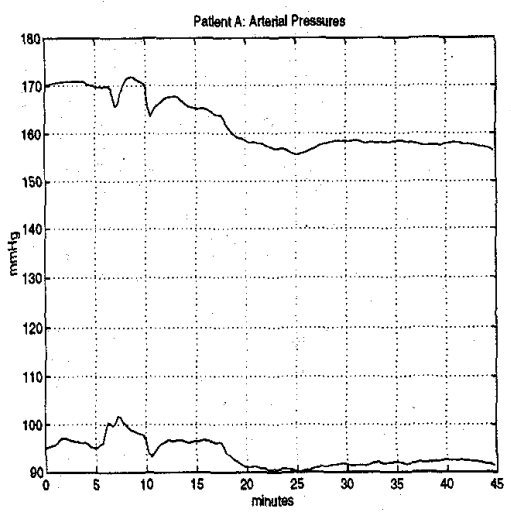

Figure 4: Patient A: Arterial Pressures

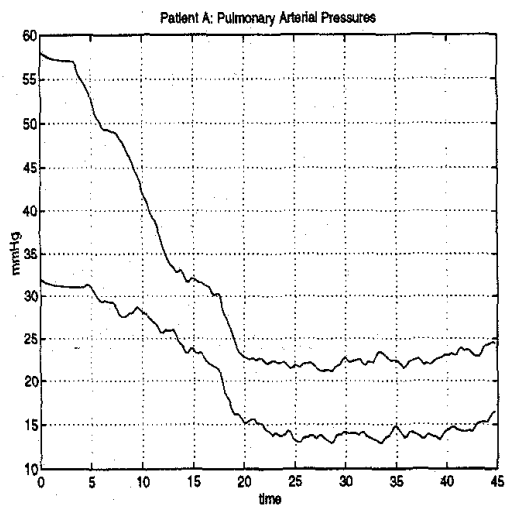

Figure 5: Patient A: Pulmonary Arterial Pressures

decreased characteristically in response to the propofol, indicating increasing anesthetic depth, but settled at values considered to be low. These low values were again believed to be due to the afore-mentioned low blood volume of Patient A.

Patient A, though having some atypical responses, provides illuminating reference data because no significant stimuli were applied during the 45 minute period recorded; this was not true in the other two subjects where many painful stimuli were applied. Because of the absence of pain it is possible to fit, using the pharmacodynamic model of Section 3, the data of Figures 4 and 5; to get close fits it is sufficient to choose the set $\mathcal{F}$ to be the one-parameter family of affine functions. In particular this data demonstrates a time delay: From Table A, a bolus (injection) was given at time 0:00 min and we see a response after approximately 4 minutes; similarly a bolus was given at 13:00 min and again a response occurs after 4 minutes. The dip in the graph at 6:00 min is due to a (large) fentanyl bolus that was administered; fentanyl is an- other anesthetic used to supplement the propofol anesthetic.

A further feature of the data obtained from $\mathrm{Pa}$ tient $A$ is that since pulmonary arterial line was initially present, we were able to obtain measurements of the pulmonary arterial pressures from the onset of anesthesia. In Patients B and C this data was not available until after 40 minutes when the pulmonary arterial line was in place, at which time a steady state response to the propofol had already been reached.

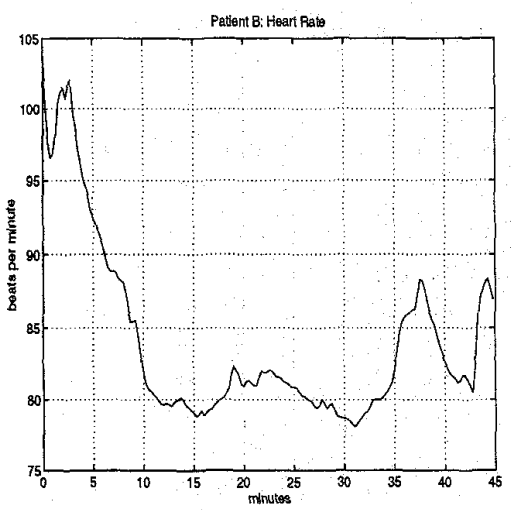

Figure 6: Patient B: Heart Rate

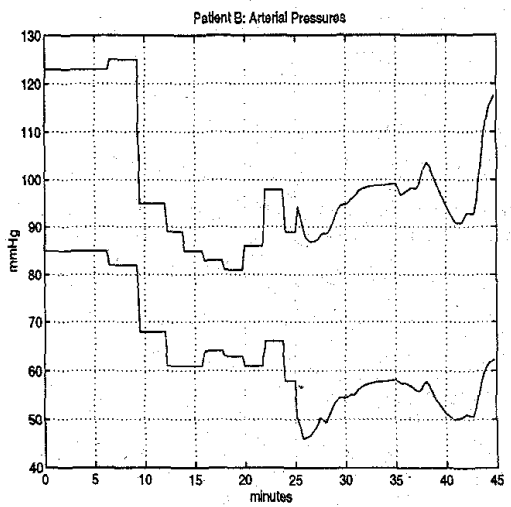

Figure 7: Patient B: Arterial Pressures

Patients $B$ and $C$ have more typical responses to the propofol, and illustrate many of the common stimulating events in surgery. The heart rate traces are shown in Figures 6 and 8. At time 0:00 both patients had yet to receive anesthetic. Once propofol was given there is a resulting small decrease in the heart rate. Before the heart rate decreases significantly there is a large increase, at $3 \mathrm{~min}$ and $8 \mathrm{~min}$ for Patients $B$ and $C$ respectively. This was caused by the painful stimulus of intubation; that is a tube was inserted into the trachea to effect mechanical 
ventilation. Once this procedure was complete the heart rates fell rapidly to approximately 20 percent below their initial values. The next significant event on the traces was caused by needle sticks, and larger catheter placements in the neck, associated with the pulmonary arterial line. This began at $35 \mathrm{~min}$ in Patient B and $22 \mathrm{~min}$ in Patient C, and produces a number of spikes as the line is guided into the pulmonary artery. The steep drop-off at $40 \mathrm{~min}$ in Figure 8 is the reaction to an injection of fentanyl.

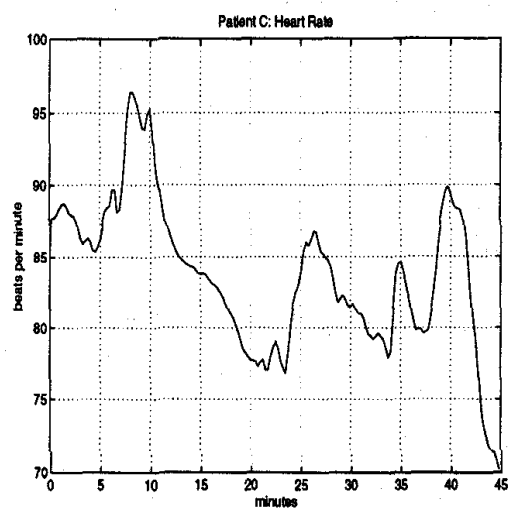

Figure 8: Patient C: Heart Rate

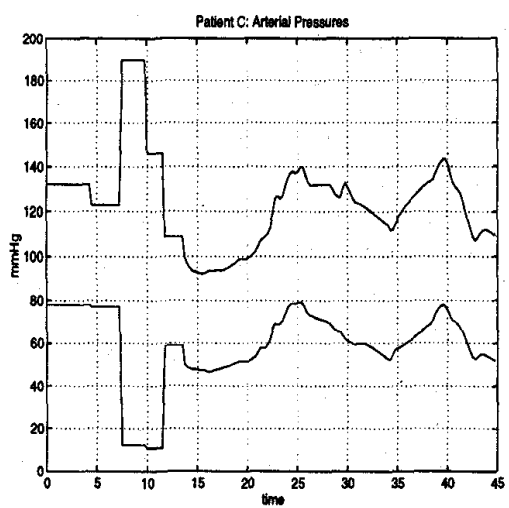

Figure 9: Patient C: Arterial Pressures

The arterial plots in Figures 7 and 9 exhibit similar responses to the anesthetic and stimuli as the heart rate plots: an increase of propofol reduces the arterial pressures and painful stimuli raises them.

\section{Conclusions}

In this paper we have identified the main areas that need to be more thoroughly researched in order to develop an automated intravenous anesthesia delivery system. After describing the basic features of clinical anesthesiology we presented a general pharmacodynamic model and (hemodynamic) data collected during surgery with the anesthetic propofol. A main objective in presenting the obtained data was to highlight the phenomena with which an automated delivery system will have to contend: large modeling uncertainties (inconsistent stimuli and patient response, unknown biological mechanisms), nonlinear patient response and poorly defined performance objectives.

We are currently examining the next stage of this research program which involves a more in depth study of the pharmacodynamic model, and the development of compatible models of stimuli. In particular the predictive abilities of these models are to be investigated.

\section{Acknowledgements}

The authors are grateful to Dr. Stan Stead of UCLA Department of Anesthesiology for the computer equipment used during data collection.

\section{References}

[1] R. Bellman, 'Topics in Pharmacokinetics, III: Repeated dosage and impulse control', Mathematical Biosciences, 12: 1-5, 1971.

[2] B.J. Ebeling, W. Muller, P. Tonner, K.T. Okkola, and H. Stoeckel, 'Adaptive feedback controlled infusion versus repetitive injections of vecuronium in patients during isoflurane anesthesia', J. of Clinical Anesthesiology, 3:181-185, 1991.

[3] N.H.G. Holford, and L.B. Sheiner, 'Understanding the dose-effect relationship: clinical application of pharmacokinetic-pharmacodynamic models', Clinical pharmacokinetics, 6:429-453, 1981.

[4] J.R. Jacobs, J.G. Reves, and P.S.A. Glass, Continuous Infusions for Maintaining Anesthesia, Little, Brown and Company, Boston, 1991.

[5] D.A. Linkens, and S.S. Hacisalihzade, 'Computer control systems and pharmacological drug administration: a survey', J. of Medical Engineering \& Technology, 14:2:41-54, March, 1990.

[6] K.T. Olkkola, and H. Schwilden, 'Quantitation of the interaction between atracurium and succinylcholine using closed-loop feedback control infusion of atracurium', Anesthesiology, 73:420-423, 1991.

[7] S.L. Schafer, L.C. Siegel, J.E. Cooke, and J.C. Scott, 'Testing computer-controlled infusion pumps by simulation', Anesthesiology, 68:261-266, 1988.

[8] H. Schwilden, H. Stoeckel, and J. Schuttler, 'Closed-loop feedback control of propofol anesthesia by quantitative EEG analysis in humans', British J. of Anesthesia, 62:290-296, 1989.

[9] N.T. Smith, M.L. Quinn, J. Flick, Y. Fukui, R. Fleming, and J.R. Coles, 'Automatic control in anesthesia: a comparison in performance between anesthetist and the machine', Anesth Analg, 63:715-722, 1984.

[10] M. White, and G.N.C. Kenny, 'Intravenous propofol anaesthesia using a computerised infusion system', Anaesthesia, 45:204-209, 1990. 